\title{
Detection of precisely edited CRISPR/Cas9 alleles through co- introduced restriction-fragment length polymorphisms
}

Chon-Hwa Tsai-Morris ${ }^{1}$, Sydney Hertafeld ${ }^{2}$, Yvonne Rosario ${ }^{1}$, James Iben ${ }^{3}$, Eric Chang $^{2}$, Ling $\mathrm{Yi}^{4}$, Steven L. Coon ${ }^{3}$, Stephen G. Kaler ${ }^{4,5}$, Ryan Dale $^{2}$ and Benjamin Feldman ${ }^{1 *}$

${ }^{1} \mathrm{NICHD}$ Zebrafish Core, Eunice Kennedy Shriver National Institute of Child Health and Human Development (NICHD), NIH, Bethesda, MD 20892, USA

${ }^{2}$ Bioinformatics and Scientific Programming Core, NICHD, NIH, Bethesda, MD 20892, USA

${ }^{3} \mathrm{NICHD}$ Molecular Genomics Core, NICHD, NIH, Bethesda, MD 20892, USA

${ }^{4}$ Section on Translational Neuroscience, NICHD, NIH, Bethesda, MD 20892, USA

${ }^{5}$ Center for Gene Therapy; Abigail Wexner Research Institute, Nationwide Children's Hospital, The Ohio State University College of Medicine; Columbus, $\mathrm{OH} 43205$

* To whom correspondence should be addressed. Tel: 001-301-443-5556; Fax: 001-301-435-6001; Email: bfeldman@mail.nih.gov

\begin{abstract}
CRISPR/Cas9 is a powerful tool for producing genomic insertions and deletions (indels) to interrogate gene function. Modified CRISPR/Cas9 protocols can produce targeted genetic changes that are more precise than indels, but founder recovery is less efficient. Focusing on producing missense mutations in zebrafish using single-stranded oligo deoxynucleotide (sSODN) donor templates, we pioneered a strategy of adding synonymous changes to create novel restriction-enzyme (RE) sites, allowing detection of rare precise edits in a modified fluorescent-PCR fragment assay. We have named this process TIARS (test for incorporation of added recognition sites). Aided by TIARS, we induced two distinct amino-acid substitutions (T979I and P1387S) in the atp7a gene among somatic tissues of CRISPR-Cas9-treated $F_{0}$ zebrafish. One of these $F_{0}$ transmitted the allele to atp $7 a^{T 979 / /+} F_{1}$ progeny, and trans-heterozygosity of this allele against a null atp7a allele causes hypopigmentation, consistent with more severe pigment deficits in zebrafish or humans carrying only null mutations in atp7a/ATP7A. Design of ssODNs with novel RE recognition sites is labor-intensive, so we developed an in silico tool, TIARS Designer, and performed bioinformatic validation indicating that TIARS should be generalizable to other genes and experimental systems that employ donor template DNA.
\end{abstract}

\section{INTRODUCTION}

Clustered regularly interspaced short palindromic repeats/Cas9 endonuclease (CRISPR/Cas9)-based mutagenesis is a popular and successful tool for generating knock-out models with disruptive insertions and deletions (indels) (1). Two essential components for CRISPR/Cas9 mutagenesis are 1) the Cas9 protein itself and 2) a gene target-specific CRISPR RNA (crRNA) that is fused or hybridized to a Cas9-specific trans-activating RNA (tracRNA). The crRNA/tracRNA hybrid is also called a synthetic guide RNA (sgRNA), or simply gRNA. These two components are delivered to cells or embryos to effect mutagenesis. Targeting and mutagenesis is restricted to genomic sequences immediately adjacent to a so-called proto-spacer adjacent motif (PAM), which for the popular Cas9 from Streptococcus pyogenes, is the sequence NGG. When the gRNA/Cas9 ribonuclear protein complex arrives at its target, it is able to create a double-strand break (DSB) upstream of the PAM site. Such DSBs may be repaired perfectly via non-homologous end joining (NHEJ), but erroneous NHEJ also frequently occurs, introducing indels, and indels that also shift the reading frame typically cause complete or partial loss of gene function.

It has more recently been discovered that, beyond random indels, one can generate precise genome edits near a target by adding a DNA repair template as a third component of the CRISPR/Cas9 mix, leading to the incorporation or copying of repair template sequence containing the edit into the 
genome with no other changes $(2,3)$. Homology-based DNA repair templates contain sequence identical to the target sequence except for desired substitutions, insertions or deletions. In the zebrafish model, which is our focus here, success has been reported for both single-stranded oligo DNA (ssODN) and double-stranded DNA (dsDNA) templates (4). Precise genome edits are reported to be generated at low rates compared to standard indels and must be discriminated from a mixture of other outcomes, namely standard NHEJ-induced indels, wild-type (WT) sequence and alleles with imprecise incorporations of template sequence. This low rate and diversity of outcomes presents challenges in 1) the analysis of injected $F_{0}$ embryos to determine whether a particular gRNA/repair template combination shows promise and 2 ) the identification of germ-line transmitting $\mathrm{F}_{0}$ adults.

Here we have focused on precise-genome editing using sSODNs to create single amino-acid changes in target genes, which has been proposed to occur via homology-directed repair (HDR) (2). Two attractive aspects of the ssODN approach are 1) the relatively short length ( $<150 \mathrm{nt})$ of the ssODNs, which can be commercially synthesized to eliminate in-house cloning or in vitro synthesis steps and 2) the correspondingly short genomic target that needs to be sequence-verified for potential divergence from the reference genome sequence. An arguable disadvantage of the ssODN approach is the lack of a fluorescent reporter gene, utilized in various dsDNA plasmid-based approaches, notably that by Hoshijima et al., which allows for in vivo screening to detect germ-line transmission from $\mathrm{F}_{0}$ founders to $F_{1}$ progeny (5). By contrast, screening when using the ssODN approach requires ex-vivo tissue samples being subjected to either direct sequencing or detection of gain or loss of a RE recognition site that was introduced by the repair template.

For standard CRISPR/Cas9 indel generation, we have learned through experience that selection of winning gRNA/Cas9 combinations is vastly aided by the ability to quickly estimate the frequency of targeted events in injected $F_{0}$ embryos. Our method of choice for this has been the CRISPR somatic tissue activity test (CRISPR-STAT), which measures the frequency of targeted indel formation in single embryos by combining fluorescent PCR and capillary electrophoresis (6). Here we describe our development of a CRISPR-STAT extension, tailored to estimate the frequency of genomic incorporation of RE recognition sites included in the SSODN template via synonymous substitutions, a method we have named TIARS (test for incorporation of added recognition sites).

The concept of TIARS emerged from our efforts to modify the atp7a gene in zebrafish. Loss of the human X-linked gene ATP7A causes Menkes disease, a disorder of copper transport that leads to death during the first few years of life if untreated $(7,8)$. Consistent with copper's essential role in pigment formation and angiogenesis, sequelae of Menkes disease include hypopigmentation and vascular abnormalities, and a current life-extending intervention combines early diagnosis with subcutaneous copper injections. The zebrafish Atp7a protein is $77 \%$ similar to the human ATP7A protein, including short stretches of near- or complete identity where cognate amino acids (AAs) are readily found. Although X-linked deficiencies cannot be modeled in zebrafish due to their lack of chromosomal sex determination, homozygosity of a null atp7a allele (named the calamity mutation, 
also called atp $7 a^{v 469}$ ) causes albinism in zebrafish larvae and death around 6 days post-fertilization (dpf) (9). In addition to Menkes disease arising from complete loss of ATP7A, a distinct human syndrome has been described for individuals carrying T994I or P1386S missense mutations in ATP7A: an isolated, adult-onset distal motor neuropathy (10-12). We initially developed TIARS to support our project to generate analogous missense alleles in zebrafish for future functional studies, i.e., to quickly monitor our success at combining Cas9, gRNA and ssODNs to induce AA coding modifications in atp7a at the cognate zebrafish positions of T979I and P1387S.

Here we report 1) our use of TIARS to detect generation of both these alleles at the somatic level, 2) germ-line transmission of the atp $7 a^{T 979 l}$ allele, which causes a pigmentation deficit in homozygous larvae and adults, 3) development of software to automate design of sSODN donor templates optimized for TIARS and 4) validation of this software by A) running it against a set of theoretical human targets and $B$ ) by high-throughput sequence analysis outcomes in the generation of a third zebrafish allele (the ryr1b gene).

\section{MATERIAL AND METHODS}

Zebrafish husbandry. EK strain wild-type (WT) zebrafish were used for most of this study, except for the high-throughput sequencing experiments, which used Tab5 strain WT zebrafish. Zebrafish were maintained in the aquatic animal facility under an Animal Use Protocol approved by the Institutional Animal Care and Use Committee (IACUC) of the Eunice Kennedy Shriver National Institute of Child and Human Development, MD, USA. All zebrafish embryos \& larvae were maintained in egg water $\left(0.006 \%\right.$ sea salts and $0.1 \%$ methylene blue) in $10 \mathrm{~cm}$ Petri dishes at $28.5^{\circ} \mathrm{C}$ for a maximum of 7 days. Larvae selected for further growth were transferred to tanks and fed no later than 6 days post fertilization (dpf).

Design and synthesis of sgRNAs. Protein sequence alignment identified T979 and P1387 of zebrafish Atp7a as cognate amino acids (AAs) of T994 and P1386S of human ATP7A. Zebrafish exon 15 for T979 and exon 22 for $\mathrm{P} 1387$ containing the $\mathrm{AA}$ codons to be modified from $\mathrm{T}$ and $\mathrm{P}$ to $\mathrm{I}$ and $\mathrm{S}$, respectively, were analyzed on Ensembl. Candidate gRNAs were identified near the target mutation sites using the NHGRI-1 (ZebrafishGenomics) Track Data Hub on the UCSC genome browser (ZV9/danRer7 assembly) and compared to more recent assemblies of the zebrafish genome with BLAST to identify any off-target matches of concern. Gene specific Alt-R CRISPR-Cas 9 crRNAs (20 mers) were purchased from IDT and co-annealed with IDT's tracrRNA (57mer) at $95^{\circ} \mathrm{C}$ for 5 min and gradually cooled to room temperature to form a functional gRNA duplex, according to the manufacturer's protocol (IDT, Skokie, II).

Single-stranded oligo mutation donor (ssODN). Asymmetric ssODNs (107 nt) complementary to the gRNA nontarget strands per Richardson et al. were designed (13). They contain the desired nonsynonymous codon mutation plus additional synonymous mutations to eliminate the PAM site and introduce two restriction enzyme (RE) sites. The two synonymous nt substitutions for creating new RE 
sites were found with the aid of re site finder (http://resitefinder.appspot.com/about) and where more than one option was available, the more frequent Danio rerio codon usage was favored according to the Kazusa Codon Usage Database (https://www.kazusa.or.jp/codon/). Putative alterations were also screened to ensure they do not affect predicted exonic splicing enhancers (ESEs) using the RESCUE-ESE Web Server http://hollywood.mit.edu/burgelab/rescue-ese/ and to ensure they do not create or eliminate any predicted microRNA targets using an additional web site (http://mamsap.it.deakin.edu.au/ amitkuma/mirna targetsnew/sequence.html) that no longer appears to be supported at the time of this submission. PAGE-purified ultramer ssODNs were purchased from IDT. The ssODN sequence for atp7a ${ }^{T 9791}$ is indicated in Fig. 1, the ssODN sequence used for atp7a ${ }^{\text {P13875 }}$ was (CATTTTAGCAGGAGGGAGGACAGCACTACAGACACAGAAGATAGAGCCATAG CAGCAGAGCCCATCCAGCTCTGAAGGACCAACCCCACAGGCATAAACACAC) and the ssODN sequence for $r y r 1 b^{14936 T}$ was (TGTTTCAGTGTTATCTGTTCCATATGTATGTGGGTGTAAGGGCCGGAG GAGGAACTGGAGATGAGATTGAAGATCCAGCTGGAGA)

Microinjection. Cas9 protein (PNA Bio, Inc.) was mixed to a final concentration of $700 \mathrm{ng} / \mathrm{ul}$ with sgRNA ( $37.5 \mathrm{ng} / \mathrm{ul})$ and ssODN ( $60 \mathrm{ng} / \mathrm{ul})$, preincubated at $37^{\circ} \mathrm{C}$ for $5 \mathrm{~min}$, centrifuged at $16,100 \mathrm{~g} 5$ min to remove any debris and $1.4 \mathrm{nl}$ was injected into the yolks of 1-4 cell embryos using the Pneumatic PicoPump system.

Preparation of embryonic and adult fin lysates for genotyping. Euthanized $5 \mathrm{dpf}$ embryos or fin biopsies from adult fish were treated with proteinase $\mathrm{K}(0.5 \mathrm{mg} / \mathrm{ml})$ in $50 \mu$ lysis buffer (20 Mm Tris$\mathrm{HCl}$ (ph 8.9), $50 \mathrm{Mm} \mathrm{KCL}, 0.3 \%$ Tween-20 and $0.3 \% \mathrm{NP}-40$ ) at $55^{\circ} \mathrm{C}$ for $2 \mathrm{hr}, 99^{\circ} \mathrm{C}$ for $5 \mathrm{~min}$ and cooled to $4^{\circ} \mathrm{C}$. Diluted lysate $(1: 10)$ was used as template for genotyping with primer sets flanking the sequence of the atp7a gene corresponding to the $107 \mathrm{nt}$ of the ssODN.

Primer design and fluorescent PCR assays. Two sets of primers [T979l editing-Forward: AGCAGTGAATGGGGAAGATG (Intron 14) and Reverse: TCCTCCCTTGATGAGGATTC (exon 15); and P1387S editing-Forward TGTTTGTCGTTTTAGTGAACC (intron 21) and reverse AAATAACCCAAATTATCATAACGGTCAC (intron 22)] were designed using Primer3Plus software to amplify 320 and 324 bp fragments, respectively, with the target $A A$ roughly in the middle of the amplicon. M13 (TGTAAAACGACGGCCAGT) and PIGtail (GTGTCTT) adapters sequences were added to forward and reverse primers respectively(6). PCR reactions contained $1 \mu \mathrm{l}$ of 1:10 diluted lysate and $5 \mu$ premixed AmpliTaq-Gold (0.3U, Life Technologies) with $0.26 \mu \mathrm{M}$ of target-specific- and an additional fluorescent forward primer: 6FAM-M13F (6FAM-TGTAAAACGACGGCCAGT)(6). In modified fluorescent PCR fragment analyses, the 6-FAM was directly linked to a forward primer with no M13 adapter sequence, and the 6FAM-M13F primer was not included. Amplification was performed on a MasterCycler, (Nexus gradient, Eppendorf) with initial denaturation at $95^{\circ} \mathrm{C}$ for 12 min, followed by 35 cycles of $\left(95^{\circ} \mathrm{C} / 30 \mathrm{~s}, 57^{\circ} \mathrm{C} / 30 \mathrm{~s}, 72^{\circ} \mathrm{C} / 30 \mathrm{~s}\right)$, and a final extension at $72^{\circ} \mathrm{C}$ for 10 min followed by a hold at $4^{\circ} \mathrm{C}$. 
Screening for site-specific mutations. Restriction fragment length polymorphism (RFLP) analysis after the fluorescent PCR reaction was used to discriminate between wild type (WT) and point mutation knock-in alleles in $\mathrm{F}_{0}$ mosaic carriers and their $\mathrm{F}_{1}$ progeny. PCR products $(5$ or $10 \mu \mathrm{l})$ were digested in the absence (control) or presence of designated REs in a total volume of $15 \mu$ for $8 \mathrm{~h}$ at $37^{\circ} \mathrm{C}$. Samples $(2 \mu \mathrm{l})$ were then mixed with $10 \mu \mathrm{l}$ of Gene Scan $400 \mathrm{HD}$ ROX dye standard $(1: 50)$ in HiDi-formamide (Life Technologies) followed by denaturation at $95^{\circ} \mathrm{C}$ for $5 \mathrm{~min}$ and a hold at $4^{\circ} \mathrm{C}$. RFLP fragments were separated in a genetic analyzer (ABI model 3130) using POP-7 polymer. Injection times were varied (23, 60 or 180 s) according to experimental design. The default injection time was was 23 seconds. Data files (.fsa) were analyzed using GeneMapper or PeakScanner software (Thermofisher).

Sequencing. For Sanger sequencing, PCR products were first treated with Exo-SapIT (Affymetrix) to eliminate unincorporated primers. Sequencing was outsourced (Macrogen USA) either (1) directly after PCR or (2) for early attempts at atp7a ${ }^{\text {T979l }}$ editing, after colony PCR using a nested reverse primer (ACTTGGTCAGGTTTCTATTTTG). Sequencing data was analyzed by Sequencher version 5.4.6. For high-throughput sequencing of $r y r 1 b$ amplicons, injected $3 \mathrm{dpf}$ embryos were lysed in groups of 10 and 1:10 dilutions were used as templates for PCR. Primers (F target-specific sequence: GCCTGACTGAGATCATGTCTTC; $R$ target-specific sequence:

ACCACACGGTAAAGTTCATACTCG) immediately flanked sequence corresponding to the ssODN, with no overlap, and included 5' Illumina adapter sequences ( $F$ adapter:

TCGTCGGCAGCGTCAGATGTGTATAAGAGACAG; R adapter

GTCTCGTGGGCTCGGAGATGTGTATAAGAGACAG). PCR amplicons (198 nt in size) were cleaned using the Qiagen QIAquick PCR purification kit according to the manufacturer's protocol and concentrations were assessed by Qubit and mixed at equimolar ratios with an amplicon distinct from ryr1b (unpublished data). Input concentration of mixed amplicons for second-round PCR-based library construction ranged from 3-10 ng/ $\mu$ l. Each library was given a unique bar code (Nextera XT library prep kit), purified, pooled with other bar-coded libraries and subjected to $2 \times 150$ bp paired-end sequencing on an Illumina MiSeq system using the MiSeq Micro v2 Reagent Kit. Demultiplexing was performed by bcl2fastq v2.20.

Computer assisted sSODN template design TIARS Designer, described below, is an open-source Python package available at https://github.com/NICHD-BSPC/tiars-designer.

\section{RESULTS}

Generation of atp7a $a^{T 9791}$ and atp7a $a^{\mathrm{P} 1387 \mathrm{~S}}$ variants. We sought to create zebrafish models for human distal motor neuropathy caused by human mutations cognate to hypothetical zebrafish mutations atp7a ${ }^{T 9791}$ and atp $7 a^{P 1387 S}$. Reports on ssODN-based CRISPR/Cas9 precise editing in zebrafish and other systems indicate that two key criteria driving efficiency are 1) minimizing the distance between the cut site (situated between position -3 and $-45^{\prime}$ to the PAM NGG) and the targeted edit, and 2) maximizing the cutting efficiency of the gRNA $(3,14)$. For the atp7 ${ }^{\text {T979l }}$ edit (a C>T change altering 
the codon threonine/ACT to isoleucine/ATT; Fig. 1B), we considered the three closest PAM-adjacent targets to the atp7a ${ }^{T 9791}$ edit site, each on the antisense strand and numbered according to their 5 ' to 3' locations: GGAGGCCTGGAAGGCGAAG (\#1, yellow), AGCGATGCAGAGGACAGTGA (\#2, green) GGTTGCGAGACCCAGAGAGC (\#3, teal). The ranking of predicted distances between the edit site and cut sites for these three candidates is: 0 nucleotides (nt; \#2) $<20 \mathrm{nt}(\# 1)<25 \mathrm{nt}(\# 3$ ). To determine cutting efficiencies of the gRNAs corresponding to these three targets, we used CRISPR-STAT as follows. gRNAs corresponding to atp7a ${ }^{\text {T979l }}$ targets 1-3 were individually co-injected into zebrafish embryos with recombinant Cas9 and individual embryos were harvested for DNA several days later. The targeted locus was PCR amplified with a primer mix that included a fluorescent label, and amplified fragments were subjected to capillary electrophoresis to reveal their migration and fluorescent intensity of each labeled PCR fragment. Cutting efficiency was calculated as the sum of non-WT peak intensities divided by the sum of all peak intensities (data not shown). Cutting scores of the three targets ranked as follows: \#1 (97\%) > \#3 (30\%) > \#2 (7\%) (Table S1). Target \#2 had the best proximity but lowest cutting score. We considered proximity to be the more important characteristic in this case because gRNAs with very high cutting scores can cause extensive somatic cell mutation. In essential genes such as atp7a, such widespread mutations are incompatible with survival and establishment of germ-line transmitting founders. Indeed, the gRNA against target \#1 fully recapitulated the lethal calamity null atp7a phenotype, including death by $6 \mathrm{dpf}$ (data not shown), and so we were regardless forced to consider scenarios of lower cutting levels.

The single strand donor template (ssODN) for precise editing was designed following the rules suggested by Richardson et al., namely with sequence complementary to the non-target strand of gRNA\#2 and with homology arms of unequal length flanking the desired modification with a bias to the $5^{\prime}$ side (13). Thus, we selected (5') 70- and (3') 37- nt homology arms (Fig. 1A underlined \& 1B). In addition to including the targeted non-synonymous $\mathrm{C}>\mathrm{T}$ change, we made three synonymous changes. First, following published recommendations aimed at reducing the chance of re-targeting and re-cutting of successful edits, we eliminated the PAM site for target \#2 with a $C>G$ change (3). Second, we introduced two additional synonymous $C>A$ changes to create novel $R E$ sites for Clal and $\mathrm{MluCl}$, in order to facilitate downstream $\mathrm{F}_{0}$ and $\mathrm{F}_{1}$ screening. The $\mathrm{C}>\mathrm{T}$ missense mutation underlying the T979l change was included in the MluCI RE site.

For atp $7 a^{P 13875}$ variant generation we used analogous reasoning and strategies, selecting a gRNA candidate with a $24 \%$ cutting score (Fig. S1) and cutting within 5 nt of the targeted edit (Fig. S1). In addition to the central non-synonymous $C C>A G$ changes (CCC/proline to AGC/serine), we introduced two additional synonymous changes ( $T>C$ and $G>T$ ) creating novel RE sites for HpyAV and $\mathrm{Hpy} 188 \mathrm{I}$. Analogous to $\mathrm{MluCl}$ for atp7a ${ }^{T 979 l}$, Hpy1881 recognizes the $\mathrm{C}>\mathrm{A}$ change that is part of the P1387S change. As a further convenience, there is a nearby endogenous Hpy1881 that could serve as an internal control for Hpy1881 activity. Because of the PAM site's registration within the coding sequence, there was no way to disrupt it with synonymous changes. Thus, we were able to find potential gRNAs and RE-site generation strategies for each of the alleles we wished to create. 
Screening for targeted atp7a edits and development of TIARS. Because of the multiplicity of mutagenic outcomes mosaically represented among somatic cells of injected $F_{0}$ embryos, direct Sanger sequencing is not a viable strategy for assessing precise editing rates. Our initial strategy for assessing the rate of atp $7 a^{T 979 l}$ edits was instead to lyse pools of injected $F_{0}$ embryos ( $n=10,5 \mathrm{dpf}$ ), PCR amplify the targeted region, and then subclone and sequence colonies. Out of 96 colonies analyzed, three had precise edits, 44 had unaltered WT sequence and 49 had various indels. We suspect, however, that these three precise edits represented false-positive artefacts. This is based on the following observations. First, further screening of 288 individual $F_{1}$ progeny from $16 F_{0}$ founders revealed template insertions only. Second, subcloning from one of these insert-carrying $F_{1}$ progeny revealed that the subcloning process itself can convert tandem repeats into precise edits, presumably due to bacterial recombination, as has been described (Fig. S2) (15).

As an alternative to subcloning, we developed TIARS by modifying CRISPR-STAT as follows. After the fluorescent PCR step, but prior to capillary electrophoresis, a portion of the PCR product is RE digested to visualize the restriction fragment length polymorphisms (RFLP) associated with precise edits that might be mosaically present in individual $F_{0}$ embryos. Our initial TIARS protocol used the cost-saving three-primer strategy of CRISPR-STAT, comprised of a "universal" 5' FAM-labeled M13 primer, an M13-tailed forward primer and a reverse primer (Fig. 2) (6). Two of $44 \mathrm{~F}_{0}$ embryos $5 \mathrm{dpf}$ after microinjecting gRNA/ssODN/Cas9 protein showed a low fluorescent RFLP signal (Fig. 2A) with the correct size (+/- $1 \mathrm{nt})$ of the predicted Clal and MluCl fragments. However, because the intensity of these signals was not far above background noise, we sought to improve the TIARS signal intensity by replacing the three-primer strategy with a two-primer strategy in which the forward primer itself carries the 5' FAM fluorophore, ensuring quantitative labeling of the amplicon. Comparing the two approaches, the fluorescent RFLP signals increased from fewer than 40 fluorescent units (FLU; Fig. $2 \mathrm{~A}$ ) to over $400 \mathrm{FLU}$ (Fig. 2B). Under this modification of TIARS, we found 2 of $31 \mathrm{~F}_{0}$ adults carrying the correct integration in fin biopsies. All told, we observed a somatic editing rate of 4 out of $75(5.3 \%)$ $F_{0}$ zebrafish for the atp7a ${ }^{T 9791}$ edit. Beyond direct labeling of the forward primer, we further augmented sensitivity by increasing the quantity of PCR product loaded onto the $A B I$ machine and the programmed injection time, the latter of which determines the fraction of sample actually loaded onto the electrophoretic capillary (Fig. S3).

TIARS on embryos injected to create atp $7 a^{\text {P1387S }}$ revealed 4 out of $41 \mathrm{Fos}_{0}(9.8 \%)$ with the predicted diagnostic pattern for Hpy1881 and HpyAV (Fig. S4). We used this same scenario to address whether phosphorothioate (PS) end-protection of the sSODN repair template might increase the rate of precise editing, as has been reported (16). However, in our hands, co-injecting a PS-protected version of the repair template yielded a lower incidence of $F_{0} s$ with the predicted diagnostic pattern: 3 out of 81 (3.8\%; Fig. S4). Unfortunately, no $F_{0}$ embryos survived from these standard ssODN and PS-ssODN experiments, likely because the gRNA was too effective and replicated the lethal phenotype of atp7a/calamity loss of function. In summary, we successfully established TIARS as a screening tool and identified two potential $F_{0}$ carriers of the atp $7 a^{T 979 l}$ mutation. 
Germ-line transmission of the atp7 ${ }^{\text {T979l }}$ allele. Our screen for germ line transmission was straightforward, as we successfully crossed a WT fish with one of the two $F_{0}$ animals that had had a diagnostic TIARS signal from its fin biopsy, and one out of the $32 F_{1}$ progeny obtained was found to be heterozygous for the modified allele according to TIARS (Fig. 3A and B), the integrity of which was then confirmed by sequencing (Fig. 3C). Per zebrafish research community convention, we gave this novel atp $7 a^{\text {T979l }}$ allele the alternate name of atp $7 a^{y 652}$, the " $y$ " indicating its institutional origin (NIH/NICHD). Applying Human Genome Variation Society rules to zebrafish, with the assistance of the Mutalyzer website (https://mutalyzer.nl), we also determined the following non-ambiguous full name for this allele: NM_001042720.1(atp7a):c.2928_2936delCTCCATCACinsATCGATAAT. For TIARS on $F_{1}$ S, we returned to our initial 3-primer version of the protocol. This was because the sensitivity required for detecting rare cells in $\mathrm{F}_{0} \mathrm{~s}$ is in fact too high when genotyping embryos that carry the same genotype in every cell. We crossed this heterozygous atp7a $a^{y 52 /+}$ animal with a heterozygous carrier of the calamity allele, atp $7 a^{v 469 /+}$, and scored and genotyped progeny, revealing a subtle hypopigmention phenotype of atp7a ${ }^{y 652 / v u 69}$ trans-heterozygous embryos (Fig. 3D-E), a phenotype that persists into adulthood (Fig. 3F-G), confirmed both by Mendelian ratios and by genotyping. Thus, with the help of TIARS, we have established a novel model for zebrafish atp7a dysfunction. Research on the general impacts of the atp $7 a^{y 652}$ allele on zebrafish health and physiology are ongoing and will be reported elsewhere.

TIARS Designer: a computer-assisted repair template design tool. Manual identification of suitable synonymous sequence alterations to generate novel RE sites is a time-consuming and errorprone process. To assist with future precise editing projects, we developed TIARS Designer, which exhaustively searches all possible RE site combinations, filters them to retain those that meet certain criteria, and ranks the remaining options for final manual selection. An overview of the computational steps is provided in Fig. 4. The inputs to the program are 1) approximately $200 \mathrm{nts}$ centered on the PAM-adjacent target of interest, 2) the PAM-adjacent target sequence, and 3) the desired nonsynonymous change.

In the first step, sequences spanning from $15 \mathrm{nt} 5$ ' to $15 \mathrm{nt} \mathrm{3'}$ of the Cas9 cut site are mutated in silico to generate the set of all possible cut site-proximal synonymous substitutions (Fig. 4). We limited the in silico synonymous substitutions to $+/-15$ nt flanking the cut site for two reasons. First, we assume that users will in general only take on such a project if they have a PAM-adjacent sequence for which the cut-site is within $15 \mathrm{nt}$ of the targeted change, as is generally recommended (3). Second, our ssODNs are typically in the $90-110 \mathrm{nt}$ range, and we wished to conserve $100 \%$ homology in the more distal portions. Depending on the identity of the amino acids, the possible number of candidate sequences containing synonymous substitutions within $15 \mathrm{nt}$ of the cut site could range from zero (in the unlikely case where all ten amino acids are methionine or tryptophan which only have a single codon) to $6^{10}$ (in the case where all ten amino acids are arginine with 6 possible codons). 
The entire set of candidate modifications is then screened against a database of commercially available REs. Each combination of RE and candidate sequence must pass a set of filters (Fig. 5) to move on to the next stage. Filter I.1 ensures each novel RE site is created by at least two synonymous substitutions. This is to limit the noise of frequent NHEJ-induced single nucleotide changes creating a RE site, a phenomenon we observed in our first attempts at TIARS with different REs (data not shown). RE sites for which nearby endogenous sites exist are attractive because the endogenous sites can serve as positive controls for RE cutting and help avoid false-negative conclusions, but the orientation and spacing of these endogenous sites needs to be carefully considered. Filter I.2 requires that no endogenous cut site occur within five nucleotides of a novel cut site so that the two fragment sizes can be reliably discerned by capillary electrophoresis. Filter I.3 ensures no endogenous RE sites flank a novel RE site on both sides. Otherwise, when PCR amplicons are digested, the labeled fluorescent primer will be separated from the diagnostic cut, regardless of which side of the amplicon it is on. Finally, filter (I.4) ensures the number of RE recognition sites are equal to the number of RE cut sites for a given RE. This is to avoid scenarios where the RE recognition site is present but not the RE cut site, which can occur with Type II REs whose cut sites are outside of the recognition site. As an additional strategy against false positive hits, we built our approach around creating two unique RE sites. Therefore the next set of filters considers pairs of REs for each candidate sequence. To be included in a RE pair for a candidate sequence, both REs must have passed the above set of filters for the sequence.

Filter II.1 ensures there are no unneeded substitutions. This avoids any superfluous nt substitutions, which in theory could diminish the rate of HDR. But an exception is made under Filter II.1 if the registration of the PAM site relative to the coding sequence is such that it cannot be disrupted by synonymous substitutions, leaving a risk of gRNA/Cas9 re-cutting of the altered allele. In these instances, a maximum of synonymous changes neutral to the novel RE design are introduced in the 10 nt 5 ' to the PAM site, the region of the PAM-adjacent target that is thought to be most important for Cas9 targeting specificity, in the hopes of inoculating the altered PAM-adjacent target against gRNA/Cas9 re-cutting (17). Filter II.2 considers endogenous sites. It has two settings: the first (harsh) looks exclusively for pairs in which each novel RE also has an endogenous site. The scenario of each RE having an endogenous site is rarer, however, and so a second Filter II.2 setting (lenient) accepts pairs where only one novel RE has an endogenous site. Filter II.3 requires that novel RE recognition sites be formed from at least one unique substitution upon which the other novel site does not also depend. This ensures that the two REs act as proxies for distinct nt substitutions. Filter II.4 demands that endogenous sites of both novel REs be on the same side of the amplicon, a requirement that allows for the convenience and cost savings of having the fluorophore on the same side of the amplicon for TIARS of either enzyme. Any remaining pairs of REs and candidate sequence that pass these filters are then considered the final candidates. The output consists of Excel spreadsheets containing the sequence, the restriction enzymes, and various statistics recorded from the filters, such as distance from RE to the Cas9 cut site, distance from RE site to the amino acid change, and other information that could be useful in ranking the hits, like codon frequency metrics. In addition to this 
spreadsheet, the tool also outputs a Word document that uses typography to encode the various features in each candidate to help with manual inspection and making the final choice for a locus.

\section{TIARS Designer Examples and Validation}

As a demonstration of TIARS Designer, Table 1 presents the numbers of candidates and solutions found for atp $7 a^{T 979 l}$ and atp $7 a^{P 1387 S}$ using the same PAM-adjacent targets that we selected and also for a new example: $r y r 1 b^{14936 T}$, a zebrafish mutation that would be cognate to the human allele $R Y R 1^{14898 T}$, which causes congenital myopathy (18). The enzyme pairs we had selected for atp7at979l and atp $7 a^{P 1387 S}$ were each one of 45 and 188 possible solutions, respectively, per TIARS Designer (Table 1). All solutions are presented as summary excel files and individual MS word documents, as shown for one of the ryr1 $1 b^{14936 T}$ solutions (Fig. 6). The excel file includes the distance of the RE cut site to the targeted amino acid change and to the Cas9 cut site and the frequency of suggested codons in zebrafish. This information can be used to rank candidate solutions according to user concerns and preferences when multiple solutions are available.

Continuing with this $r y r 1 b^{14936 T}$ example, we injected zebrafish embryos with an oligo containing these changes, pooled and lysed samples ( $n=10,3 \mathrm{dpf})$, PCR amplified the targeted region and performed high-throughput sequencing as part of a larger study (manuscript under preparation). Analysis of the combined total reads from two such samples, as well as combined total reads from three negative controls in which Cas9 and the gRNA but no oligo were injected, indicates the following. First, that ryr $1 b^{14936 T}$ has somatic rates of perfect edits representing $3.2 \%$ out the total reads and $16.6 \%$ out of those WT and perfectly edited reads that were not otherwise altered (6020 MUT reads out of 186870 Total Overall reads and 6020 MUT reads out of 39,262 MUT + WT reads, respectively; Fig. 7A "+" and Table 2.). The overall high rates of "OTHER" reads (Fig. 7A and Table 2), meaning reads that were altered in length or had non-synonymous changes beyond the targeted AA change, likely reflects a combination of mutagenesis due to erroneous NHEJ events, non-homologous inclusion of sSODN sequence and PCR/High-throughput sequencing-introduced errors, as has been observed in other high-throughput sequencing analyses of zebrafish HDR experiments (19). Second, our analysis indicates a $97.3 \%$ coincidence of reads ( 5850 of 6020 MUT reads) bearing the desired edit and the combination of novel REs and no other confounding changes to RE sites. This indicates that TIARS would theoretically have served as an excellent proxy for the desired edit (Fig. 7B and Table 2). Stated otherwise, there were only $2.7 \%$ candidate false-negative reads (170 of 6020 MUT reads) where TIARS would theoretically have failed due to a lack of diagnostic RE site creation or other changes to the diagnostic pattern. Also rare $(0.06 \%$; 19 out of 33,242 total WT reads) were falsepositive scenarios in which one or the other of the diagnostic novel RE sites was present but not the desired edit (Fig. 7C and Table 2). This low rate of false-positive outcomes indicates the requirement for hypothetical RE sites to be created from a minimum of two synonymous mutations (Fig. 5, filter 1.i) is sufficiently stringent. 
To dig in further, we next asked whether this "two or greater" synonymous mutations per novel RE site rule (Fig. 5, filter 1.i) is not only sufficient, but also necessary to minimize false positives. To do this, we considered novel RE sites that would have been created if only one of the two requisite nt changes had occurred. As a comparison against the twin (AGCTGG to gGCcGG) substitutions used to create a novel Haelll site ( $\underline{G G C C}$ ), we considered the frequency with which an adjacent Hpall site (CCGG) was formed that can also arise from a single nt substitution (AGCTGG to AGCcGG).

Similarly, the frequency of a novel site arising from only one of the substitutions intrinsic to creating a Bpml site (CTGGAG via twin ATGGAG to ctGGAG substitutions) was calculated, though in this case an imaginary RE site was queried. Although the number of hypothetical false-positive WT reads increased from 19 to 144 in these single nt substitution scenarios (Table 2), the overall false-positive rate only increased from $0.06 \%$ to $0.4 \%$ (144 out of 33242 reads; Table 2 ), suggesting that an option for RE site creation via single nt changes could be a useful addition to future versions of TIARS Designer.

\section{DISCUSSION}

A challenge of genome editing in the zebrafish model is the long feedback loop between the execution of a genome-editing perturbation and the identification of alleles carrying desired edits. For other model organisms with shorter life spans, such as $C$. elegans (3), it is sufficient to wait a few weeks (compared to 4 months for zebrafish) for the next generation and assess $F_{1}$ progeny for germ line transmission. For mice, embryonic-stem cells allow for pre-screening of edits of interest. Our experience has been that the ability to assess somatic rates of desired edits in injected $\mathrm{F}_{0}$ embryos is critical for quality control and for efficiently comparing methods and variables. To this end, we routinely use the CRISPR-STAT fluorescent PCR-based approach for assessing the ability of gRNAs to produce standard indels (6).

We initially considered two methods for examining precise edits: subcloning of PCR amplicons and high-throughput sequencing. Our exploration of the former led to a waste of time due to a falsepositive artifact. We ultimately figured out that subcloning amplicons containing tandem repeats can produce altered sequences due to bacterial deletions or expansions of the repeat (15). Given that tandem repeats are a common outcome of Cas9/HDR procedures (19), we now avoid subcloning to evaluate Cas9/HDR outcomes and recommend that others use caution as well.

High-throughput sequencing is certainly a viable approach for assessing the somatic mutational spectrum among $\mathrm{F}_{0}$ embryos, and one that we use ourselves (manuscript in preparation). Here, we have presented our development of TIARS, an alternative approach that combines RFLP with CRISPR-STAT. While it is true that costly DNA analysis equipment is required for both TIARS and high-throughput sequencing, a growing number of companies perform these analyses for a fee and TIARS may be more time- or cost-efficient for small investigator-led projects than high-throughput sequencing. For instance, TIARS allows for a two-day turnaround from tissue harvest to data analysis, enabling researchers with limited husbandry space to quickly prioritize groups of animals of 
interest, such as $\mathrm{F}_{0}$ larvae from the same microinjected cohort as sampled specimens showing promising rates of somatic edits. Turnaround for high-throughput sequencing is longer, requiring at least one week, necessitating husbandry of a larger zebrafish population while awaiting results. Other pros and cons of TIARS vs. high-throughput-sequencing for detection of somatic edits have not been fully assessed, including the question of which method is more sensitive. The typical high-throughput sequencing error rate (below which no novel edits can reliably be detected) can reach $0.1 \%(20)$, whereas we have not yet determined the TIARS limits of detection.

Our group's standard CRISPR/Cas9 practices were applied to this project and certain approaches to HDR editing were also adopted on faith. Among our standard practices is the use of commercially synthetized gRNAs. Standard laboratory synthesis of gRNAs requires a 5'GG pair for optimal yields (21). Certain workarounds, such as modification of gRNAs with 5' NG or GN termini to 5'GG have been shown to be effective (22). Nonetheless, commercially synthesized gRNAs have no sequence constraints at all, allowing researchers to test any PAM-adjacent sequence of choice, such as the 5'TT gRNA we selected for the atp7a $a^{P 1387 S}$ project.

Without having run any in-house quantitative comparisons against other sSODN designs, we have followed on faith the recommendation that ssODNs be complementary to the non-target strand and that the homology arms asymmetrically flank the predicted Cas 9 cut site with a bias to the 5' side (13). Also on faith, we have followed the principle of including PAM-site or gRNA-binding disruptions in our ssODNs, as theoretical inoculation against re-cutting of edited alleles by persisting gRNA/Cas9 (3). We did run a small-scale test of the proposal that phosophorothioate-end protection of ssODNs increases HDR editing efficiency as has been reported (16), with no supporting evidence in our hands, though we do not claim our single counter-example comprises a critical evaluation of the proposal.

Zebrafish atp7a is an essential gene (9), which rendered it impossible for us to apply yet another common recommendation for increasing HDR editing efficiency: that the gRNA cutting efficiency be maximized (3). The essential role of atp7a was indeed a constraint for us, even preventing it seemed, our successful use of a gRNA with a cutting efficiency as low as $24 \%$ on a scale that often exceeds $90 \%$. The one gRNA that worked for us to establish germ-line transmission had the very low cutting efficiency of $7 \%$. Our success using such an inefficient gRNA suggests that cutting efficiency can be sacrificed in certain contexts. The fact that this gRNA's cut site was precisely at the location of the desired edit, another recommended HDR-editing design principle, seems likely to have contributed to our success (3). Of course genome-editing strategies that can alter coding sequence using gRNAs that target non-coding sequence and thus leave the protein unperturbed in most failed editing scenarios, such as the method of Hoshijima et al., offer an alternative workaround for editing essential genes (5). 
The exercise of manually searching for synonymous changes to create novel REs is both time consuming and error prone. Furthermore, the programming and implementation of TIARS Designer to solve this problem soon made us realize how far we had been from considering all sSODN design possibilities in any kind of unbiased or comprehensive manner. We subsequently have used this tool for a number of unpublished studies. Our foray into computational analysis of CRISPR/Cas9-based HDR editing also helped us more definitively establish that synonymous changes serve as excellent proxies for their non-synonymously changed neighbors, with extremely low false positive or false negative rates. These analyses further suggested that a lower bar of filtration allowing creation of novel RE sites via only one rather than two nt substitutions could be adequate. The question of whether supernumerary synonymous changes might in and of themselves affect HDR rates, is an outstanding question that we plan to experimentally address in future studies.

In closing, having conceived and developed TIARS and TIARS Designer as new tools in the genomeediting arsenal, we hope they will be useful to other researchers engaged in ssODN-based HDR gene editing projects and related approaches.

\section{DATA AVAILABILITY}

TIARS Designer, an open-source Python package available at https://github.com/NICHD-BSPC/tiarsdesigner

\section{SUPPLEMENTARY DATA}

Supplementary Table S1 and Figures S1-S4 have been provided in a separate file.

\section{ACKNOWLEDGEMENT}

Thanks to Tianwei Li and Joseph R. Zoeller for assistance with high-throughput sequencing. Thanks to the aquatics staff of the NIH Central Aquatics facility and members of the Research Animal Management Branch for their excellent overall support and humanitarian care and oversight of the zebrafish used in this research. Thanks to Blake R. Carrington, Daniel A. Castranova, Andrew E. Davis, Tokunbor A. Lawal and Bradley S. Toms for technical support and intellectual input.

\section{FUNDING}

This work was supported by the following National Institutes of Health intramural research programs: ZIC HD008921-09 Gene Function, Expression and Regulation in Zebrafish (NICHD; BF); Z01 HD008892 Mechanisms of Motor Neuron Disease (NICHD; SGK); NIH Bench-to-Bedside Award Mechanisms and Treatment of Motor Neuron Disease Associated with Copper Metabolism Defects (NIH Clinical Center; SGK, BF). Funding for open access charge: NICHD/ZIC HD008921-09

\section{CONFLICT OF INTEREST}

None

\section{REFERENCES}


1. Doudna, J.A. and Charpentier, E. (2014) Genome editing. The new frontier of genome engineering with CRISPR-Cas9. Science, 346, 1258096.

2. Zhang, J.H., Adikaram, P., Pandey, M., Genis, A. and Simonds, W.F. (2016) Optimization of genome editing through CRISPR-Cas9 engineering. Bioengineered, 7, 166-174.

3. Paix, A., Folkmann, A., Rasoloson, D. and Seydoux, G. (2015) High Efficiency, HomologyDirected Genome Editing in Caenorhabditis elegans Using CRISPR-Cas9 Ribonucleoprotein Complexes. Genetics, 201, 47-54.

4. $\quad$ Albadri, S., De Santis, F., Di Donato, V. and Del Bene, F. (2017) In Jaenisch, R., Zhang, F. and Gage, F. (eds.), Genome Editing in Neurosciences, Cham (CH), pp. 41-49.

5. Hoshijima, K., Jurynec, M.J. and Grunwald, D.J. (2016) Precise genome editing by homologous recombination. Methods Cell Biol, 135, 121-147.

6. Carrington, B., Varshney, G.K., Burgess, S.M. and Sood, R. (2015) CRISPR-STAT: an easy and reliable PCR-based method to evaluate target-specific sgRNA activity. Nucleic Acids Res, 43, e157.

7. Kaler, S.G. (2011) ATP7A-related copper transport diseases-emerging concepts and future trends. Nat Rev Neurol, 7, 15-29.

8. Kaler, S.G., Holmes, C.S., Goldstein, D.S., Tang, J., Godwin, S.C., Donsante, A., Liew, C.J., Sato, S. and Patronas, N. (2008) Neonatal diagnosis and treatment of Menkes disease. N Engl J Med, 358, 605-614.

9. Madsen, E.C. and Gitlin, J.D. (2008) Zebrafish mutants calamity and catastrophe define critical pathways of gene-nutrient interactions in developmental copper metabolism. PLoS Genet, 4, e1000261.

10. Kennerson, M.L., Nicholson, G.A., Kaler, S.G., Kowalski, B., Mercer, J.F., Tang, J., Llanos, R.M., Chu, S., Takata, R.I., Speck-Martins, C.E. et al. (2010) Missense mutations in the copper transporter gene ATP7A cause X-linked distal hereditary motor neuropathy. Am J Hum Genet, 86, 343-352.

11. Yi, L., Donsante, A., Kennerson, M.L., Mercer, J.F., Garbern, J.Y. and Kaler, S.G. (2012) Altered intracellular localization and valosin-containing protein (p97 VCP) interaction underlie ATP7A-related distal motor neuropathy. Hum Mol Genet, 21, 1794-1807.

12. Yi, L. and Kaler, S.G. (2015) Direct interactions of adaptor protein complexes 1 and 2 with the copper transporter ATP7A mediate its anterograde and retrograde trafficking. Hum $\mathrm{Mol}$ Genet, 24, 2411-2425.

13. Richardson, C.D., Ray, G.J., DeWitt, M.A., Curie, G.L. and Corn, J.E. (2016) Enhancing homology-directed genome editing by catalytically active and inactive CRISPR-Cas9 using asymmetric donor DNA. Nat Biotechnol, 34, 339-344.

14. Burg, L., Palmer, N., Kikhi, K., Miroshnik, E.S., Rueckert, H., Gaddy, E., MacPherson Cunningham, C., Mattonet, K., Lai, S.L., Marin-Juez, R. et al. (2018) Conditional mutagenesis by oligonucleotide-mediated integration of loxP sites in zebrafish. PLoS Genet, 14, e1007754.

15. Bzymek, M. and Lovett, S.T. (2001) Instability of repetitive DNA sequences: the role of replication in multiple mechanisms. Proc Natl Acad Sci U S A, 98, 8319-8325.

16. Liang, X., Potter, J., Kumar, S., Ravinder, N. and Chesnut, J.D. (2017) Enhanced CRISPR/Cas9mediated precise genome editing by improved design and delivery of gRNA, Cas 9 nuclease, and donor DNA. J Biotechnol, 241, 136-146.

17. Kuscu, C., Arslan, S., Singh, R., Thorpe, J. and Adli, M. (2014) Genome-wide analysis reveals characteristics of off-target sites bound by the Cas9 endonuclease. Nat Biotechnol, 32, 677683.

18. Robinson, R., Carpenter, D., Shaw, M.A., Halsall, J. and Hopkins, P. (2006) Mutations in RYR1 in malignant hyperthermia and central core disease. Hum Mutat, 27, 977-989.

19. Boel, A., De Saffel, H., Steyaert, W., Callewaert, B., De Paepe, A., Coucke, P.J. and Willaert, A. (2018) CRISPR/Cas9-mediated homology-directed repair by ssODNs in zebrafish induces 
complex mutational patterns resulting from genomic integration of repair-template fragments. Dis Model Mech, 11.

20. Clement, K., Hsu, J.Y., Canver, M.C., Joung, J.K. and Pinello, L. (2020) Technologies and Computational Analysis Strategies for CRISPR Applications. Mol Cell, 79, 11-29.

21. Varshney, G.K., Carrington, B., Pei, W., Bishop, K., Chen, Z., Fan, C., Xu, L., Jones, M., LaFave, M.C., Ledin, J. et al. (2016) A high-throughput functional genomics workflow based on CRISPR/Cas9-mediated targeted mutagenesis in zebrafish. Nat Protoc, 11, 2357-2375.

22. Moreno-Mateos, M.A., Vejnar, C.E., Beaudoin, J.D., Fernandez, J.P., Mis, E.K., Khokha, M.K. and Giraldez, A.J. (2015) CRISPRscan: designing highly efficient sgRNAs for CRISPR-Cas9 targeting in vivo. Nat Methods, 12, 982-988.

\section{TABLE AND FIGURES LEGENDS}

Table 1. TIARS Designer pass/fail statistics for the complete set of synonymous substitution candidates (within a $30 \mathrm{nt}$ window centered on the cut site) for atp7a ${ }^{T 9791}$, atp7a $a^{\mathrm{P} 1387 \mathrm{~S}}$ and ryr1b $b^{14936 T}$.

Table 2. Inventory of high-throughput reads among $F_{0}$ embryos targeted for creation of an ryr1 $\mathbf{b}^{14936 T}$ allele. Reads were initially classified as either encoding the unaltered WT AA sequence (WT), encoding the desired AA substitution only (MUT) or encoding additional AA changes (OTHER). Further classifications considered various combinations of the following features: presence (CC) vs. absence (no CC) of the targeted codon change; presence of both the novel Bpml and HaellI RE sites (both Rsites)(All desired changes); absence of the endogenous Bpml site (no endog Rsite); presence of only one or the other of the novel Bpml or Haelll sites (no novel Rsite)(only novel Rsite)(off target Rsite)(apparent novel Rsite); and absence of both novel Bmpl or Haelll sites (neither Rsite). The same categories were also applied and inventoried for the Hpall RE and an imaginary New Enzyme with RE sites that can be created by single nt substitutions, as opposed to the Bpml and Haelll RE sites that require two nt substitutions in the context of $r y r 1 b^{14936 T}$.

\section{Figure 1. Knock-in strategy for generating the atp7a ${ }^{\text {T979l }}$ allele}

A. Genomic sequence of the zebrafish atp7a locus (exon 15) with the upstream intron (14-15) sequence italicized. Sequences corresponding to candidate gRNAs, each on the opposite strand, are highlighted (\#1 in yellow, \#2 in green and \#3 in teal), and neighboring (opposite strand) PAM sequences are in bold. The \#2 gRNA was selected for the editing project. The blue arrow indicates the T979 target codon and the full genomic sequence corresponding to the selected SsODN is underlined.

B. Schematic representation of the knock-in and detection strategy. Atp7a exon 15, black box. The ssODN donor template (red) has the identical sequence as the targeted strand (i.e., the reverse complement of the [sense] sequences shown in this figure) with the exception of four key changes (vertical arrows in detail), namely: one non-synonymous change (yellow star) and three synonymous 
changes (green stars) that together eliminate the PAM site (pink oval in schematic) and introduce Clal and $\mathrm{MluCl}$ sites. Yellow lightning bolt on schematic indicates the double-strand cut site by Cas 9 as directed by the gRNA (horizontal dashed arrow). Location of primers for detection (see Fig. 2) indicated as horizontal arrows. Note that primers flank all sequence corresponding to the single strand oligo donor, reducing the risk of amplifying the sSODN sequence from any other location or context.

Figure 2. Fo screening strategy for ssODN-directed changes by RE digestion of fluorescent

\section{PCR prior to fragment analysis}

Top panel: Schematic representation of original $(A)$ versus modified $(B)$ fluorescent PCR fragment analysis. Original approach of PCR reaction with three primers, 5'FAM-M13 and M13 tailed Forward and Reverse primers. Modified PCR reaction with two primers, 5'FAM directly linked to the forward primer and reverse primer. Predicted amplicon sizes using 3-primer/or 2-primer strategies (18 nt difference due to presence/absence of M13 sequence on 6FAM-labeled strand) are indicated in the corner box.

Lower panel: Fluorescent PCR was performed using the original method (A) and modified approaches (B). Results are shown are for an adult fin biopsy. The PCR product was digested in the absence (Control) or presence of REs Clal or MluCl prior to analysis. Migration distance and flluorescent intensity of the 5' labeled strand spanning from the 6-FAM label to the other end of the amplicon or the 5' RE cut site was measured on an ABI 3130 Capillary Electrophoresis unit. The uncut amplicon size was either $A=320 \mathrm{nt}$ or $\mathrm{B}=302 \mathrm{nt}$ depending on the presence or absence of the $18 \mathrm{nt} \mathrm{M} 13$ sequence. A) Pre-digestion with Clal or MluCl led to the appearance of novel low-intensity peaks below 40 fluorescent units (flu) close to the predicted sizes for a precisely edited allele. We attribute the discrepancies of $+5 \mathrm{nt}$ for the Clal fragment ( $205 \mathrm{nt}$ observed vs. $200 \mathrm{nt}$ predicted) and $+7 \mathrm{nt}$ for the $\mathrm{MluCl}$ fragment (211 observed vs. $204 \mathrm{nt}$ predicted) to sequence-dependent variance of fragments from molecular standards, as frequently observed on the ABI 3130, with relatively larger effects on relatively smaller fragments. B) Direct labeling of the 5' primer increased sensitivity more than 10-fold, with the novel peaks now showing intensities $>400$ flu (compare $A$ and $B$ ). No other REdependent peaks of this magnitude were observed. As with the low-intensity peaks in $A$ ), there was a variance of $+5 \mathrm{nt}$ ( $187 \mathrm{nt}$ observed vs. 182 predicted) and $+7 \mathrm{nt}$ (193 nt observed vs. $186 \mathrm{nt}$ predicted), all observations and predictions being reduced by $18 \mathrm{nt}$, in accordance with the elimination of the $18 \mathrm{nt}$ M13 sequence from the modified 6FAM-labeled 5' primer.

\section{Figure 3. Germ-line transmission and sequence verification of the atp7a ${ }^{T 9791}$ edit}

A-B. Germline transmission was demonstrated by heterozygosity of the allele of interest among $F_{1}$ embryo progeny from an $\mathrm{F}_{0}$ female candidate carrier (based on previous fin biopsy results as per Fig. 2) crossed with a WT male. ABI electrophoretic traces showing wild-type alleles at $320 \mathrm{nt}$ and preciseedited allele after RE digestion with Clal at $205 \mathrm{nt}$ (200 predicted) and $\mathrm{MluCl}$ at $211 \mathrm{nt}$ (204 nt predicted). C. Sequence verification. Arrows indicate mixed WT/edited-allele peaks at each of the four targeted positions, as well as mixed peaks at two single-nucleotide polymorphisms (SNPs) known to be present in the EK zebrafish strain. 
D-G. Hypopigmented phenotype of atp7a ${ }^{y 652 / v u 69}$ larval and adult fish. D-E. Representatives of the two phenotypes seen among $5 \mathrm{dpf}$ progeny of a cross between an atp $7 a^{y 652 /+}$ female and an atp $7 a^{\text {vu69/+ }}$ male. $75.7 \%$ (143 of 189 ) had the standard WT phenotype (D), characterized by very dark eyes (arrow) and melanocytes (bracket). 24.3\% had a subtle but discernibly hypopigmented phenotype (E) with lighter eyes (arrow) and melanocytes (bracket). Subsets of these dark and light fish were raised in separate tanks and the hypopigmented phenotype persisted (compare $\mathrm{G}$ with $\mathrm{F}$ ) at 3 months postfertilization. Genotyping of fin biopsies from the hypopigmented cohort revealed that $100 \%$ (16/16: $\chi^{2}$ $P=1.2 \times 10^{-5}$ ) had the atp $7 a^{y 652 / v u 69}$ genotype, whereas fin biopsies from the dark cohort yielded representatives of the other three genotypes, namely atp7a $a^{v u 69 /+}(5 / 22)$, atp $7 a^{y 652 /+}(8 / 22)$ and atp $7 a^{+/+}$ (9/22), but none of the atp7a ${ }^{y 652 / v 669}$ genotype $\left(0 / 22: \chi^{2} P=1.2 \times 10^{-5}=0.012\right)$.

\section{Figure 4. TIARS Designer flow chart.}

Repair template candidates are generated as follows. The data entered is the locus sequence with the desired non-synonymous change and the location of the predicted cut site (between position -3 and -4 of the NGG PAM for Cas9). The first computational step generates the set of sequences carrying all possible combinations of synonymous changes within $15 \mathrm{nt}$ of the predicted cut site. Each of these sequences is then subjected to two sequential sets of pass-fail queries: the Single Enzyme filter and the Enzyme Pair filter, as detailed in Fig. 5.

Figure 5. TIARS Designer filter criteria. Repair template candidates have to pass four tests each for the Single Enzyme filter (I) and the Enzyme Pair filter (II).

\section{Figure 6. Sample outputs of TIARS Designer (example: ryr1 $b^{14936}$ )}

Top: Summary list (Microsoft Excel) enabling a ranking of repair template candidates according to criteria provided in the column headers.

Bottom: Individual pages (Microsoft Word) for each candidate with detailed annotations.

\section{Fig. 7. Sequence inventory of $r y r 1 b^{14936 T}$ edits and other outcomes}

among $F_{0}$ (injected) embryos. Total reads from combined independent pools of ten $3 \mathrm{dpf}$ Tab5 embryos each that had been injected at the $0-4$ cell stage ( $0 \mathrm{dpf})$ with a combination of Cas 9 , the selected ryr1 $b^{14936}$-targeting gRNA (grey highlight, Fig. 6) and the ssODN ("+", two independent pools) or without the ssODN (“"-", four independent pools). See Table 2 for further details. 


\section{Table 1. TIARS Designer Pass/Fail statistics

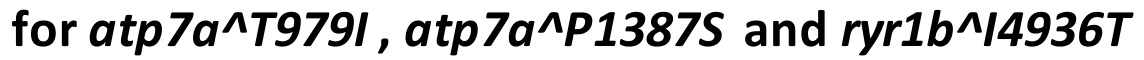

\begin{tabular}{|l|l|l|l|l|l|l|l|}
\hline Sample & $\begin{array}{l}\text { no } \\
\text { sandwiched } \\
\text { cutsites }\end{array}$ & $\begin{array}{l}\text { no } \\
\text { endogenous } \\
\text { cutsites } \\
\text { within Nnt of } \\
\text { novel cutsite }\end{array}$ & $\begin{array}{l}\text { novel site } \\
\text { depends on } \\
\text { at least two } \\
\text { substitutions }\end{array}$ & $\begin{array}{l}\text { num } \\
\text { recognition } \\
\text { sites equals } \\
\text { num } \\
\text { cutsites }\end{array}$ & $\begin{array}{l}\text { no unneeded } \\
\text { substitutions }\end{array}$ & $\begin{array}{l}\text { Total } \\
\text { Candidates } \\
\text { before SE } \\
\text { filtering }\end{array}$ & $\begin{array}{l}\text { Total } \\
\text { Candidates } \\
\text { after SE } \\
\text { filtering }\end{array}$ \\
\hline Atp7a P1368S & 62.5 & 70.32 & 27.49 & 81.3 & 8.75 & 526344 & 10136 \\
\hline Atp1a T979| & 60.2 & 66.27 & 25.12 & 70 & 0.06 & 498042 & 48 \\
\hline Ryr1b 14936T & 71.08 & 76.43 & 37.47 & 84.86 & 14.1 & 5133504 & 212304 \\
\hline
\end{tabular}

Enzyme Pair Filters

\begin{tabular}{|l|l|l|l|l|l|l|l|}
\hline Samples & $\begin{array}{l}\text { at least one } \\
\text { has } \\
\text { endogenous } \\
\text { site }\end{array}$ & $\begin{array}{l}\text { both have } \\
\text { endogenous } \\
\text { site }\end{array}$ & $\begin{array}{l}\text { compatible } \\
\text { polarity }\end{array}$ & $\begin{array}{l}\text { both } \\
\text { Renzymes } \\
\text { depend on } \\
\text { at least one } \\
\text { unique } \\
\text { substitution }\end{array}$ & $\begin{array}{l}\text { no unneeded } \\
\text { substitutions }\end{array}$ & $\begin{array}{l}\text { Total } \\
\text { Candidates } \\
\text { before EP } \\
\text { filtering }\end{array}$ & $\begin{array}{l}\text { Total } \\
\text { Candidates } \\
\text { after EP } \\
\text { filtering }\end{array}$ \\
\hline Atp7a P1368S & 37.68 & 2.36 & 98.06 & 47.94 & 19.54 & 158176 & 188 \\
\hline Atp7a T979I & NA & NA & 100 & 45.8 & 0.16 & 38256 & 45 \\
\hline Ryr1b 14936T & 13.76 & 1.21 & 99.54 & 58.4 & 18.43 & 4073400 & 1040 \\
\hline
\end{tabular}




\section{Table 2. Inventory of High-Throughput Reads Among F0 Embryos Targeted for ryr1b^/4936T}

\begin{tabular}{|l|r|r|r|r|}
\hline & BpmI & Haelll & Hpall & New Enzyme \\
\hline All desired changes (MUT) & 5991 & 5879 & 5877 & 5862 \\
\hline CC|no novel Rsite (MUT) & 18 & 141 & 135 & 135 \\
\hline CC|no endog Rsite (MUT) & 5 & $\mathrm{NA}$ & $\mathrm{NA}$ & $\mathrm{NA}$ \\
\hline CC|neither Rsite (MUT) & 0 & $\mathrm{NA}$ & $\mathrm{NA}$ & $\mathrm{NA}$ \\
\hline no CC|both Rsites (WT) & 0 & $\mathrm{NA}$ & $\mathrm{NA}$ & $\mathrm{NA}$ \\
\hline no CC| only novel Rsite (WT) & 0 & 11 & 11 & 11 \\
\hline no CC|neither Rsite (WT) & 40 & $\mathrm{NA}$ & $\mathrm{NA}$ & $\mathrm{NA}$ \\
\hline CC| off target Rsite (MUT) & 6 & 0 & 8 & 23 \\
\hline no CC| off target Rsite (WT) & 8 & 0 & 64 & 58 \\
\hline off target CC|apparent novel Rsite (OTHER) & 0 & 0 & 0 & 0 \\
\hline category sum & 6068 & 6031 & 6095 & 6089 \\
\hline total WT & 33242 & 33242 & 33242 & 33242 \\
\hline total MUT & 6020 & 6020 & 6020 & 6020 \\
\hline total OTHER & 147608 & 147608 & 147608 & 147608 \\
\hline total overall & 186870 & 186870 & 186870 & 186870 \\
\hline
\end{tabular}


Fig. 1. Knock-in Strategy for atp7a ${ }^{T 979 I}$

A.

TATTGAATGGAGAATGTGTTTCTCTGCAG GGT TAT GAT AAG AGT ATC TCT GAA GCG GAG GCT $\# 1$

GTC ATC CGC TTC GCC TTC CAG GCC TCC ATC ACT GTC CTC TGC ATC GCT TGC CCC TGC TCT

CTG GGT CTC GCAACC CCC ACA

$T^{\star 979}$

B.

Primer-F1 $\rightarrow$

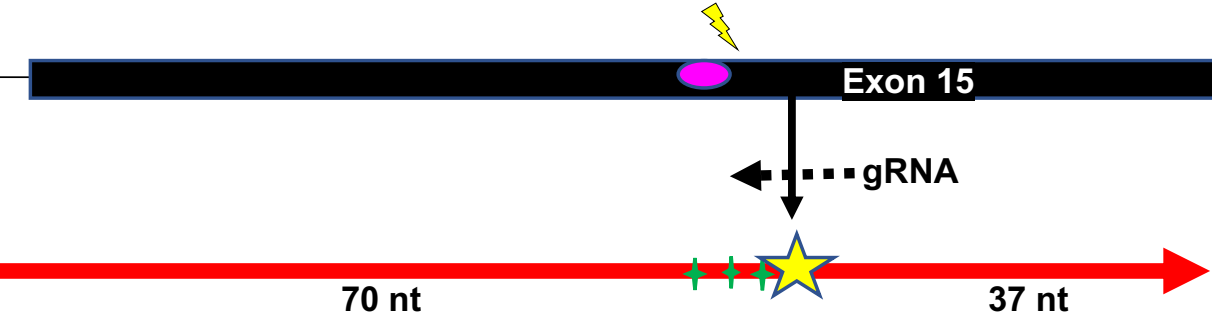

$\rightleftarrows$ Primer-R1

ssDonor

$70 \mathrm{nt}$

$37 \mathrm{nt}$

Point mutation

PAM site

$+\quad$ Synonymous change

Cas9 cut site 
Fig. 2. $F_{0}$ Screening with a Combination of Fluorescent PCR Fragment Analysis and RFLP

(2/44 injected embryos, 2/31 adult fin biopsies)

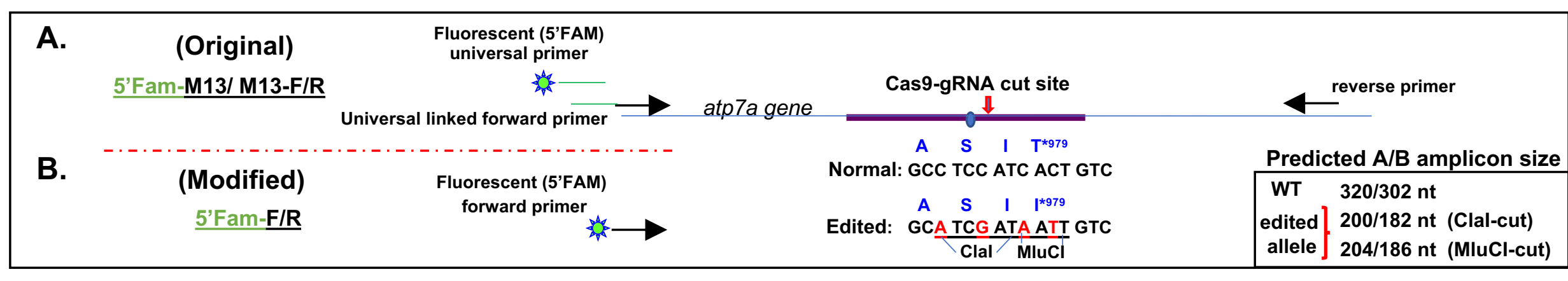

A.

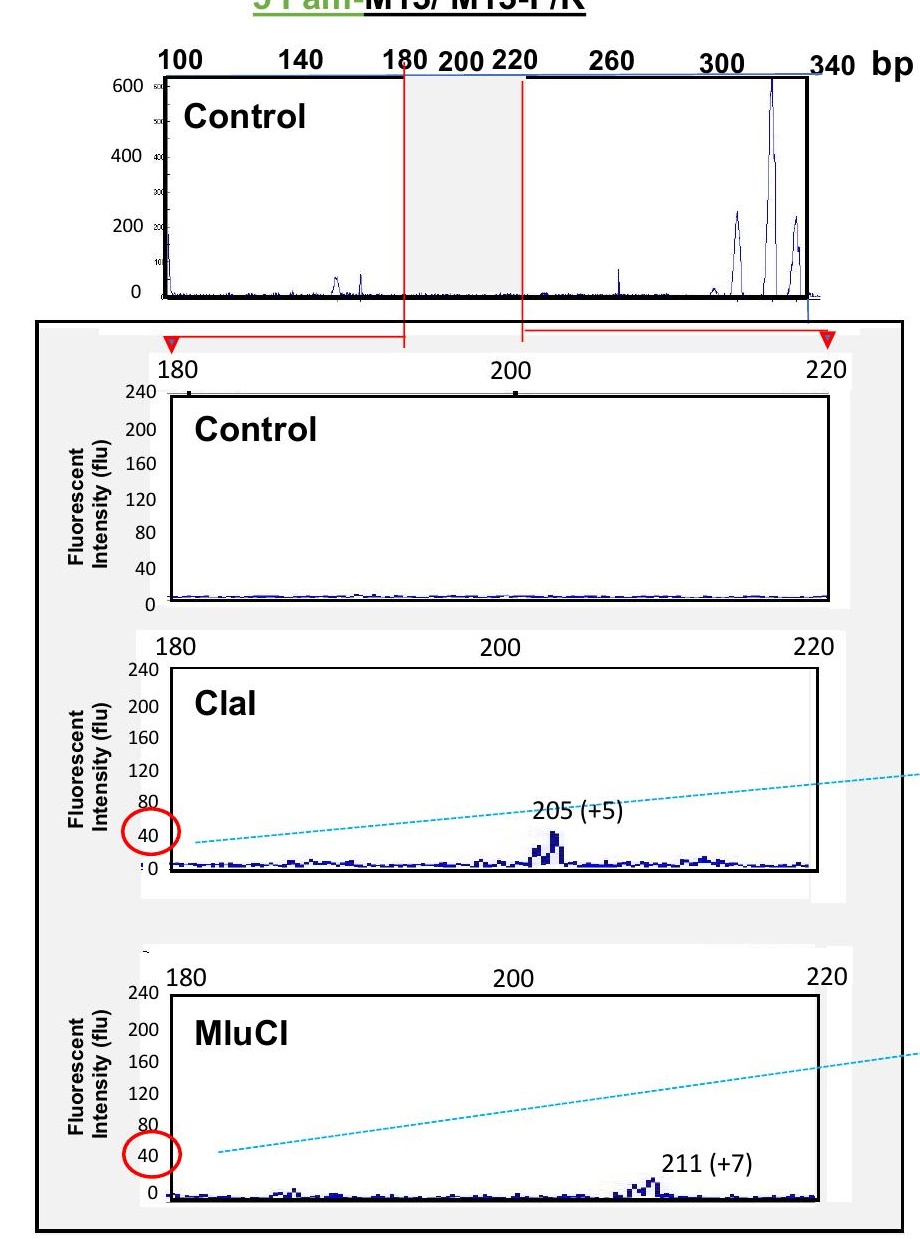

B.

$\underline{5^{\prime} \mathrm{Fam}-\mathrm{F} / \mathbf{R}}$
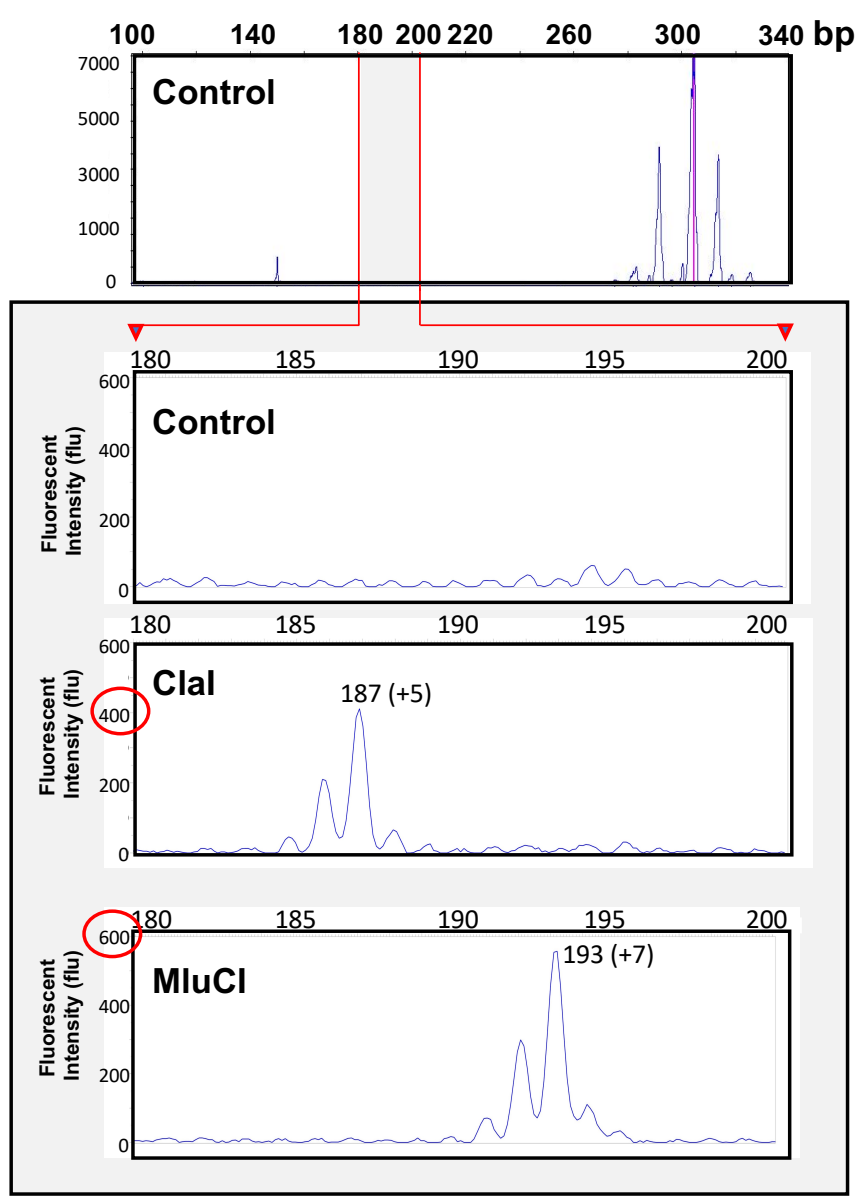
Fig. 3. Germ-Line Transmission of the atp7a ${ }^{T 979 l}$ allele (atp7a ${ }^{y 652}$ )
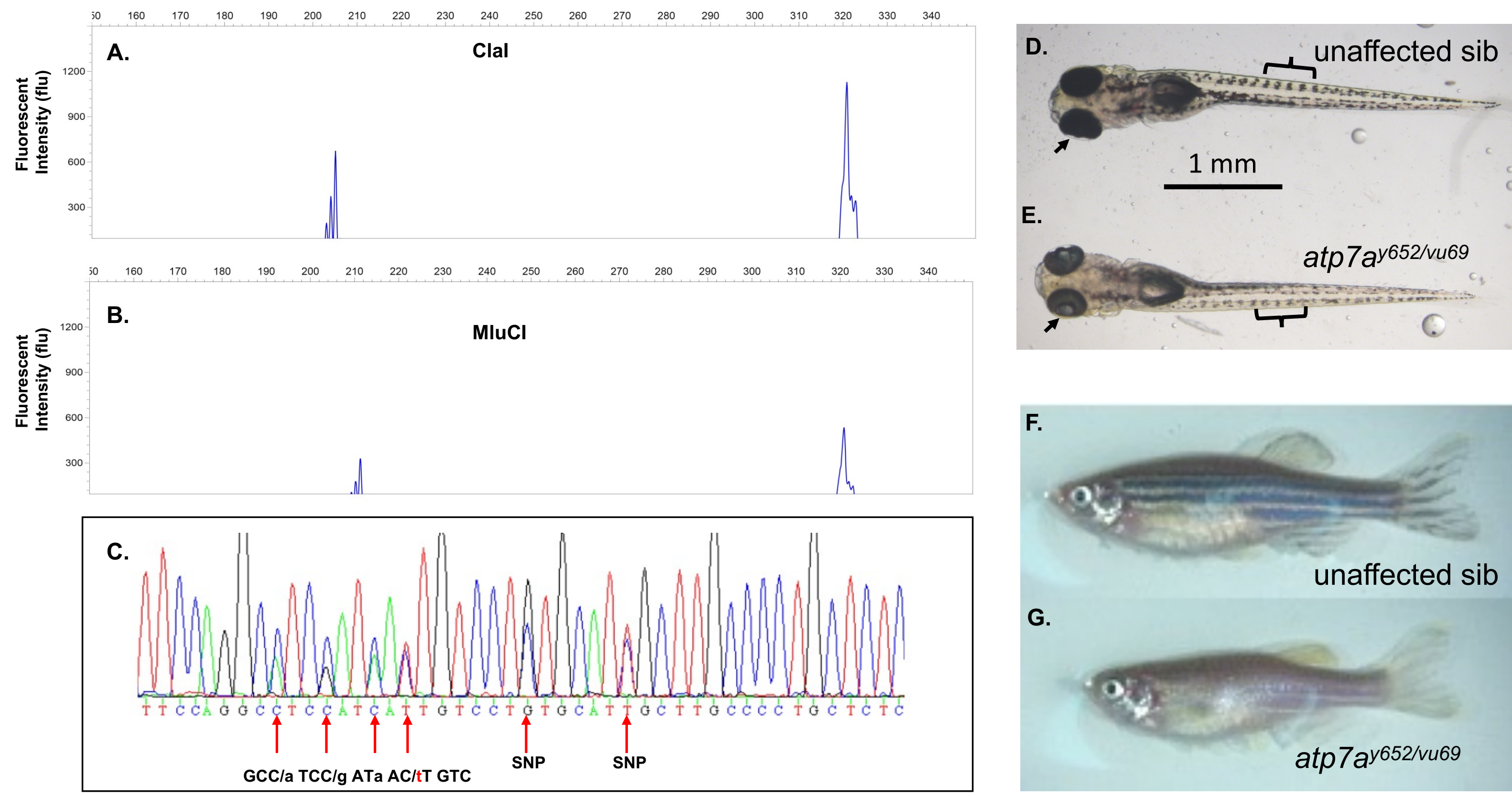


\section{Fig. 4. TIARS Designer Flow Chart}

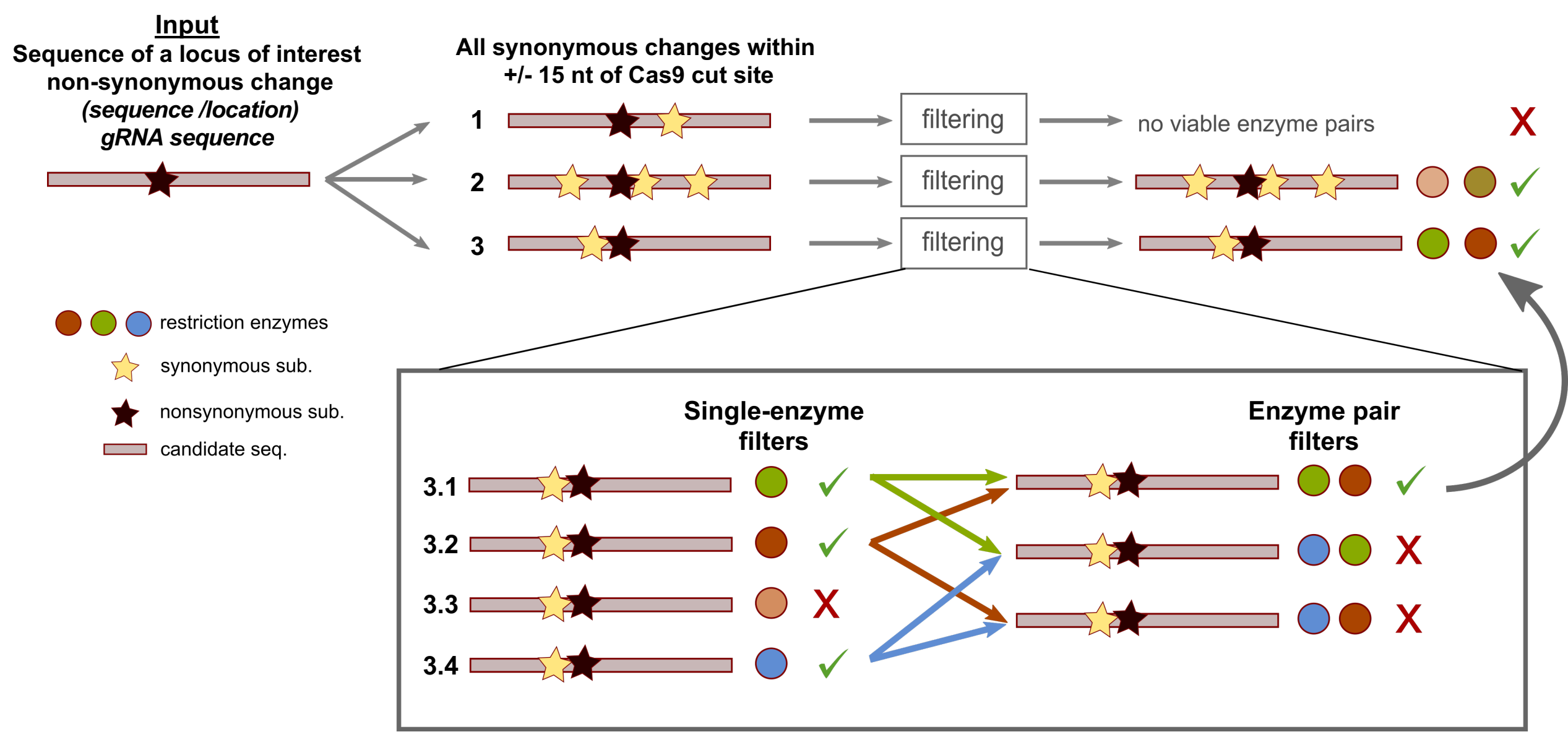




\section{Single enzyme filters}

$\underset{A_{\text {nov }}}{A_{\overline{e n d}}} \quad \begin{gathered}\text { Candidate repair oligo with } \\ \text { two synonymous substitutions }\end{gathered}$

nov: Novel RE for Enzyme A (or B)

(created by substitutions)

end: Endogenous site

for enzyme A (or B)
1. Novel site depends on at least two substitutions

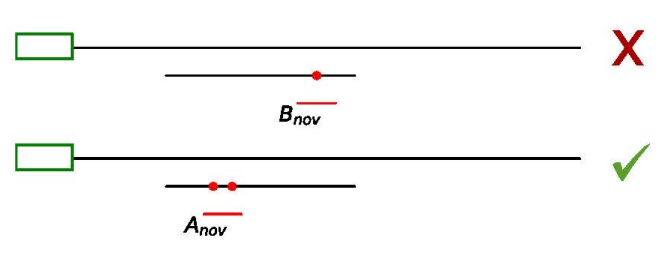

\section{Enzyme Pair filters}

1. No unnecessary substitutions PAM site disrupted?

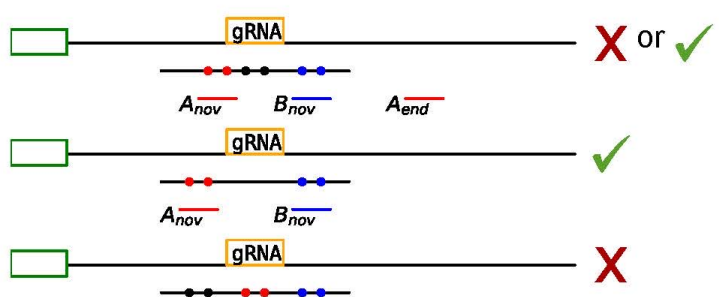

2. No endogenous cut sites within 5 nt of novel cutsite

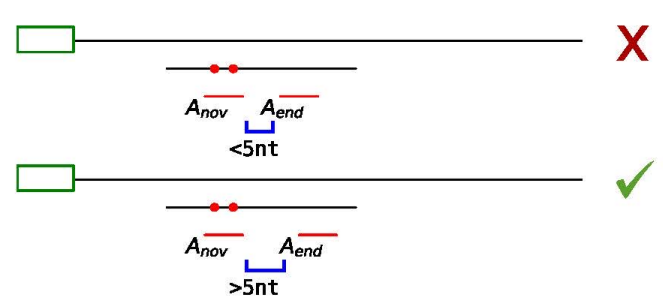

2. One or both enzymes have endogenous sites

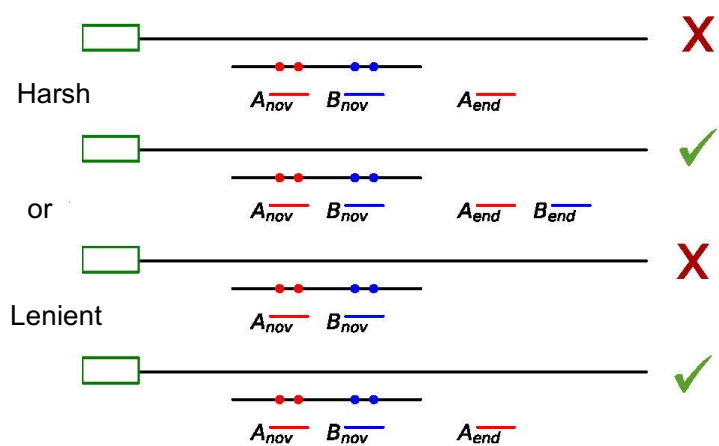

3. No sandwiched cut sites

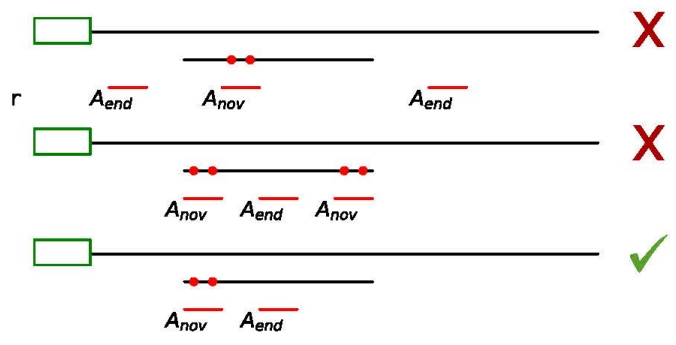

4. \# of recognition sites = \# of cut sites

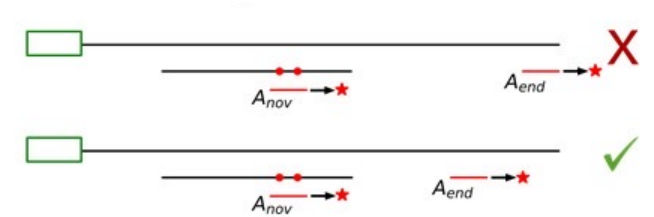

3. Both enzymes depend on at least one unique substitution

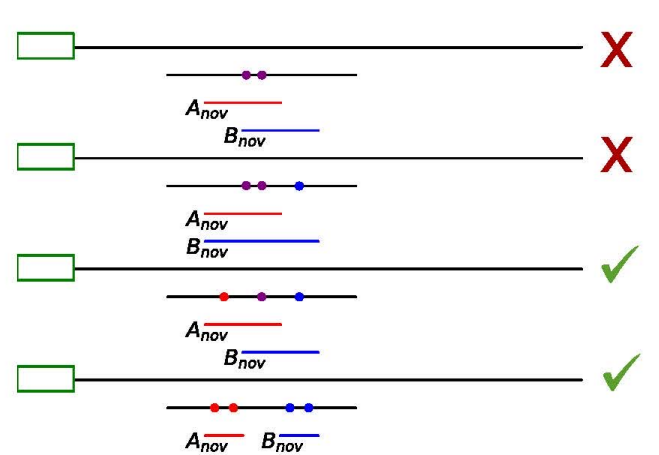

4. Compatible polarity of endogenous sites

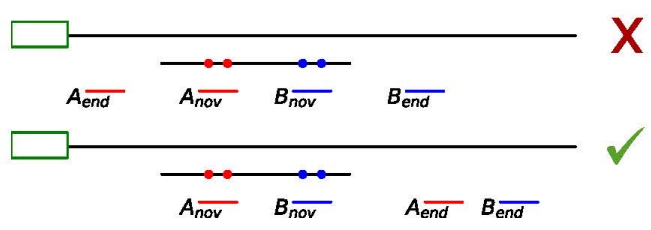


Fig. 6. Sample outputs of TIARS Designer

\section{Ex: Ryr1b $b^{14898 T}$ (ATA to ACT) Subset of the 1040 that passed out of 4073400 candidates}

\begin{tabular}{|c|c|c|c|c|c|c|c|c|c|c|}
\hline Rank & Option \# & Renzyme pair & $\begin{array}{c}\text { Renzyme } \\
\text { A avg } \\
\text { distance } \\
\text { from cas9 } \\
\text { cut }\end{array}$ & $\begin{array}{c}\text { RenzymeA } \\
\text { cutsite } \\
\text { distance } \\
\text { outside } \\
\text { Rsite }\end{array}$ & $\begin{array}{l}\text { RenzymeA } \\
\text { distance } \\
\text { to Amino } \\
\text { Acid } \\
\text { change }\end{array}$ & $\begin{array}{l}\text { Renzyme } \\
\text { B avg } \\
\text { distance } \\
\text { from cas9 } \\
\text { cut }\end{array}$ & $\begin{array}{c}\text { RenzymeB } \\
\text { cutsite } \\
\text { distance } \\
\text { outside } \\
\text { Rsite }\end{array}$ & $\begin{array}{l}\text { RenzymeB } \\
\text { distance to } \\
\text { Amino } \\
\text { Acid } \\
\text { change }\end{array}$ & $\begin{array}{l}\text { codon } \\
\text { frequency } \\
\text { differences }\end{array}$ & $\begin{array}{c}\text { overall } \\
\text { avg } \\
\text { distanc } \\
\text { e from } \\
\text { cas9 } \\
\text { cut }\end{array}$ \\
\hline 1 & 8 & HaellI and Mnll & 10 & 0 & 11 & 21 & 0 & 22 & 18.7 & 15.5 \\
\hline 2 & 9 & Haelll and Mnll & 10 & 0 & 11 & 21 & 0 & 22 & 22.2 & 15.5 \\
\hline 5 & 5 & Haelll and Bpml & 10 & 0 & 11 & 24 & 0 & 20 & 16.2 & 17 \\
\hline 6 & 6 & Haelll and Bpml & 10 & 0 & 11 & 24 & 0 & 20 & 18.9 & 17 \\
\hline 7 & 7 & Haelll and Bpml & 10 & 0 & 11 & 24 & 0 & 20 & 19 & 17 \\
\hline 8 & 11 & HpyCH4IV and Bpml & 14 & 0 & 15 & 24 & 0 & 20 & 18.8 & 19 \\
\hline 9 & 12 & HpyCH4IV and Bpml & 14 & 0 & 15 & 24 & 0 & 20 & 19.4 & 19 \\
\hline 14 & 2 & Bpml and Mnll & 24 & 17 & 20 & 21 & 17 & 22 & 27.3 & 22.5 \\
\hline 15 & 3 & Bpml and $\mathrm{Mnll}$ & 24 & 17 & 20 & 21 & 17 & 22 & 27.5 & 22.5 \\
\hline
\end{tabular}

Option 7:

HaeIII (GG^_CC) and BpmI (CTGGAGNNNNNNNNNNNNNN_NN^N)

ACAAACCTTCTACATTTGGTTCAGTTACATTTTCTCTCACTTCACGCCTGACTGAGATCATGTCTTCTGTTTCAGTGTTATCTGTTCCATA TGTATGTGGGTGTCCGGGCCGGTGGGGGCACTGGAGATGAGATTGAAGATCCAGCTGGAGACGAGTATGAACTTTACCGTGTGGTTTTTG ACATCACCTTCTTCTTTTTTGTTATTGTCATCCTGCTGGCCATCATTCAAGGTGAGGAAAACGTTTGTTTCATGGCAGGGTTTTTTTCTCA $\mathrm{G}^{* *} \mathrm{FAM}$

\section{PAM}

Amino acid change

Renzyme A recognition site

Renzyme $B$ recognition site

$\wedge=$ cut on $(+)$ strand

= cut on (-) strand

base pair substitution 
Fig. 7. Inventory of $r y r 1 b^{14936 T}$ reads among $F_{0}$ embryos.

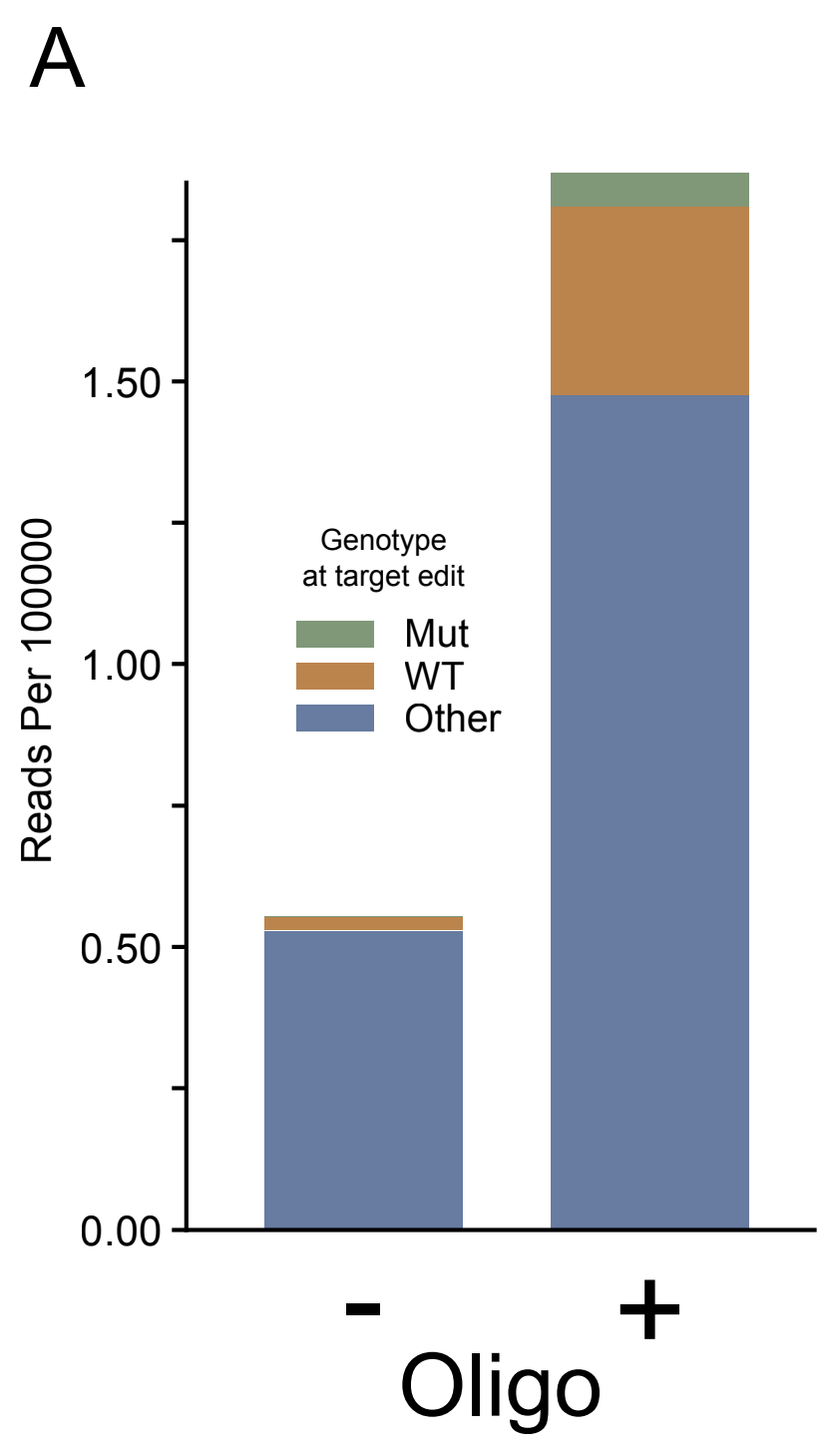

B

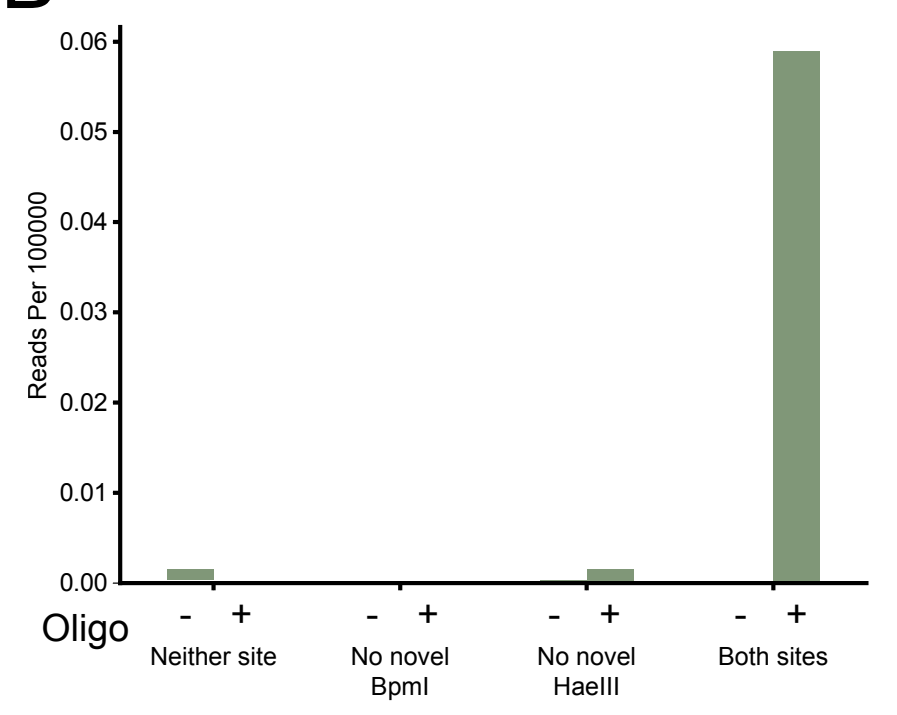

C

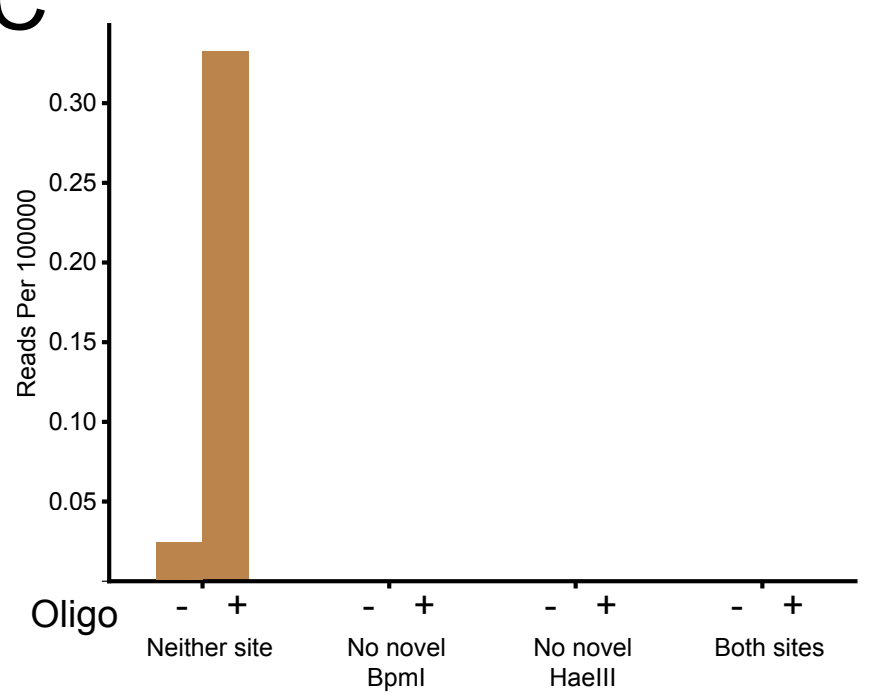

